\title{
A morfometria de ostras determina a riqueza e abundância do biofouling?
}

\author{
Does oyster morphometry determine the richness and abundance of biofouling? \\ ¿La morfometría de la ostra determina la riqueza y abundancia del bioincrustante?
}

Recebido: 29/01/2021 | Revisado: 05/02/2021 | Aceito: 14/02/2021 | Publicado: 21/02/2021

Mara Rúbia Ferreira Barros
ORCID: https://orcid.org/0000-0003-1354-7550
Universidade Federal do Pará, Brasil
E-mail: eng.p.marabarros@gmail.com
Weverton John Pinheiro dos Santos
ORCID: https://orcid.org/0000-0001-8413-5081
Universidade Federal Rural da Amazônia, Brasil
E-mail: weverton john@hotmail.com
Marko Herrmann
ORCID: https://orcid.org/0000-0001-9891-6700
Universidade Federal Rural da Amazônia, Brasil
E-mail: marko.herrmann@gmx.de
Wagner Cesar Rosa dos Santos
ORCID: https://orcid.org/0000-0001-8172-9917
E-mail: wagpesca@yahoo.com.br
Rafael Anaisce das Chagas
Centro de Pesquisa e Gestão de Recursos Pesqueiros do Litoral Norte, Brasil
ORCID: https://orcid.org/0000-0002-1555-6154
Universidade Federal do Pará, Brasil
E-mail: rafaelanaisce@hotmail.com

\begin{abstract}
Resumo
O biofouling é um dos principais problemas que acometem cultivos de ostras. Neste estudo, objetivou-se testar a hipótese de que a riqueza e a abundância do macrobentos que compõe o biofouling estão associadas à área de superfície da ostra Crassostrea tulipa cultivada no rio Urindeua, Pará. Para tanto, realizaram-se nove coletas entre maio de 2016 e abril de 2017, amostrando-se 30 ostras/mês em um cultivo de ostras localizado no litoral amazônico. Primeiramente, retirou-se o biofouling da superfície das ostras, realizando posteriormente a triagem, quantificação e identificação taxonômica dos organismos. Para testar possíveis diferenças entre ostras de diferentes tamanhos com relação aos parâmetros riqueza e abundância do biofouling, aplicou-se uma ANOVA one-way, seguida de um teste post hoc de Tukey. Os resultados deste estudo confirmam a hipótese de que a riqueza de biofouling está associada à superfície da ostra, indicando que ostras maiores apresentam um maior número de espécies quando comparadas às menores. No entanto, refutou a hipótese quando se relacionou a abundância.
\end{abstract}

Palavras-chave: Abundância; Ostra-do-mangue; Macrobentos; Riqueza.

\begin{abstract}
Biofouling is one of the main problems that affect oyster crops. In this study, it was tested the hypothesis that the richness and abundance of macrobentos that make up biofouling are associated with the surface area of the oyster Crassostrea tulipa cultivated in the Urindeua River, Pará. For this, nine collections were carried out between May 2016 and April 2017, showing 30 oysters/month in an oyster crop located on the Amazon coast. First, biofouling of the oyster surface was removed, and the classification, quantification and taxonomic identification of the organisms were subsequently carried out. To test possible differences between oysters of different sizes in relation to the parameters richness and abundance of biofouling, a ANOVA one-way was applied, used a Tukey's post hoc test. The results of this study confirm the hypothesis that the richness of biofouling is associated with the oyster surface, indicating that larger oysters have a higher number of species when compared to smaller ones. However, it refuted the hypothesis when abundance was related.
\end{abstract}

Keywords: Abundance; Mangrove oyster; Macrobenthos; Richness.

\section{Resumen}

El biofouling es uno de los principales problemas que afectan a los cultivos de ostras. En este estudio, se probó la hipótesis de que la riqueza y abundancia de macrobentos que componen el bioincrustante se asocia con la superficie de la ostra Crassostrea tulipa cultivada en el río Urindeua, Pará. Para ello, nueve colecciones se llevaron a cabo entre mayo de 2016 y abril de 2017, mostrando 30 ostras/mes en un cultivo de ostras situado en la costa amazónica. En primer lugar, se eliminó el bioincrustante de la superficie de la ostra, y posteriormente se llevó a cabo la clasificación, 
cuantificación e identificación taxonómica de los organismos. Para probar posibles diferencias entre ostras de diferentes tamaños en relación con la riqueza de parámetros y la abundancia del bioincrustante, se aplicó un ANOVA one-way, seguido de una prueba de teste post hoc Tukey. Los resultados de este estudio confirman la hipótesis de que la riqueza del bioincrustante está asociada con la superficie de la ostra, lo que indica que las ostras más grandes tienen un mayor número de especies en comparación con las más pequeñas. Sin embargo, refutó la hipótesis cuando la abundancia estaba relacionada.

Palabras clave: Abundancia; Ostra de mangle; Macrobentos; Riqueza.

\section{Introdução}

Denomina-se biofouling à formação de depósitos biológicos sobre superfícies artificiais (e.g., cascos de embarcações e instalações portuárias) ou superfícies naturais (e.g., conchas de bivalves) (Bannister et al., 2019; Chagas et al., 2018). Os organismos que assentam/colonizam o biofouling desempenham um importante papel ecológico nos ecossistemas aquáticos, devido à posição que estes ocupam dentro da cadeia alimentar, pois participam intensamente da decomposição da matéria orgânica e da ciclagem de nutrientes (Nybakken \& Bertness, 2004).

O tipo de substrato define a estrutura do habitat, assim como a estrutura da comunidade do macrobentos e, consequentemente, a formação do biofouling, que pode se diferenciar facilmente entre superfícies naturais e artificiais (Connell \& Glasby, 1998; Vale et al., 2020). A aquicultura, com destaque à malacocultura, possibilita uma importante função no ecossistema, oferecendo um habitat alternativo para a macroepifauna nativa, mesmo em pequenas escalas (Marenghi \& Ozbay, 2010). Os bivalves dispostos em condições de cultivos exercem funções similares aqueles que habitam ambientes naturais (Chagas et al., 2018; Shumway et al., 2003; Sievers et al., 2014). Esses cultivos propiciam a formação de uma estrutura capaz de manter outros organismos vivendo associados às conchas das ostras, em vários níveis de simbiose (Bannister et al., 2019; Sievers et al., 2019).

Todavia, dependendo da proporção desta simbiose, prejuízos ao cultivo são acometidos, causando uma queda na produtividade (Bannister et al., 2019; Sievers et al., 2014), com proporções que variam de acordo com a localização geográfica, as espécies de moluscos, o habitat e o método de cultura (Lacoste \& Gaertner-Mazouni, 2014). Há casos em que, a simples presença de alguns organismos (e.g., poliquetas perfuradoras) inviabiliza-o cultivo, devido ao consumo de recursos e tempo na tentativa de reduzi-los, o que pode elevar excessivamente os custos de sua produção (Bannister et al., 2019; Lacoste \& GaertnerMazouni, 2014; Sievers et al., 2014).

No Brasil, os estudos relacionados à composição do biofouling em cultivo de bivalves concentram-se na região Sul, com destaque aos estados de Paraná e Santa Catarina, sendo este último responsável pela quase totalidade da produção nacional de moluscos bivalves. Os estudos realizados nesses estados abordaram apenas a composição do biofouling associado a superfície dos bivalves, sem analisar características morfológicas ou morfométricas das conchas. Dentre as espécies estudadas estão: ostras Crassostrea spp. (Frigotto, 2011; Pinto, 2007), vieiras Nodipecten nodosus (Linnaeus, 1758) (Carraro, 2008; Macedo, 2012) e mexilhão Perna perna (Linnaeus, 1758) (Leite, 2007; Macedo et al., 2012; Marenzi \& Branco, 2006). Já na região Sudeste, Camargo (2011) caracterizou o biofouling em cultivos de P. perna no litoral dos estados de São Paulo e Rio de Janeiro. Na região Nordeste do Brasil, no estado da Bahia, o biofouling foi caracterizado em cultivos de Mytella guyanensis (Lamarck, 1819) (Souza et al., 2007). E na região Norte, no estado do Pará, Chagas et al. (2018) descreveram a composição do biofouling na superfície de ostras Crassostrea sp.

Neste estudo objetivou-se testar a hipótese de que tanto a riqueza quanto a abundância de biofouling estariam associadas à superfície da concha da ostra-do-mangue Crassostrea tulipa (Lamarck, 1819) - nome atualizado da ostra Crassostrea gasar (Deshayes, 1830), segundo World Register of Marine Species (www.marinespecies.org) - em um estuário amazônico, localizado no estado do Pará, região Norte do Brasil. 


\section{Metodologia}

\section{Local de estudo}

A área de estudo delimita-se no cultivo de ostras da Associação dos Agricultores, Pecuaristas e Aquicultores ASAPAQ, situado rio Urindeua, localizado na vila de Santo Antônio de Urindeua (Figura 1), município de Salinópolis, estado do Pará, Amazônia Oriental, Norte do Brasil. Na ASAPAQ cultiva-se a ostra-do-mangue C. tulipa através de sementes adquiridas na comunidade de Nova Olinda, município de Augusto Corrêa. O sistema de cultivo utilizado na ASAPAQ é do tipo suspenso, usando lanternas e travesseiros.

Figura 1. Mapa de localização do cultivo de ostras da Associação dos Agricultores, Pecuaristas e Aquicultores - ASAPAQ, situado no rio Urindeua, localizado na vila de Santo Antônio de Urindeua, região Norte do Brasil.
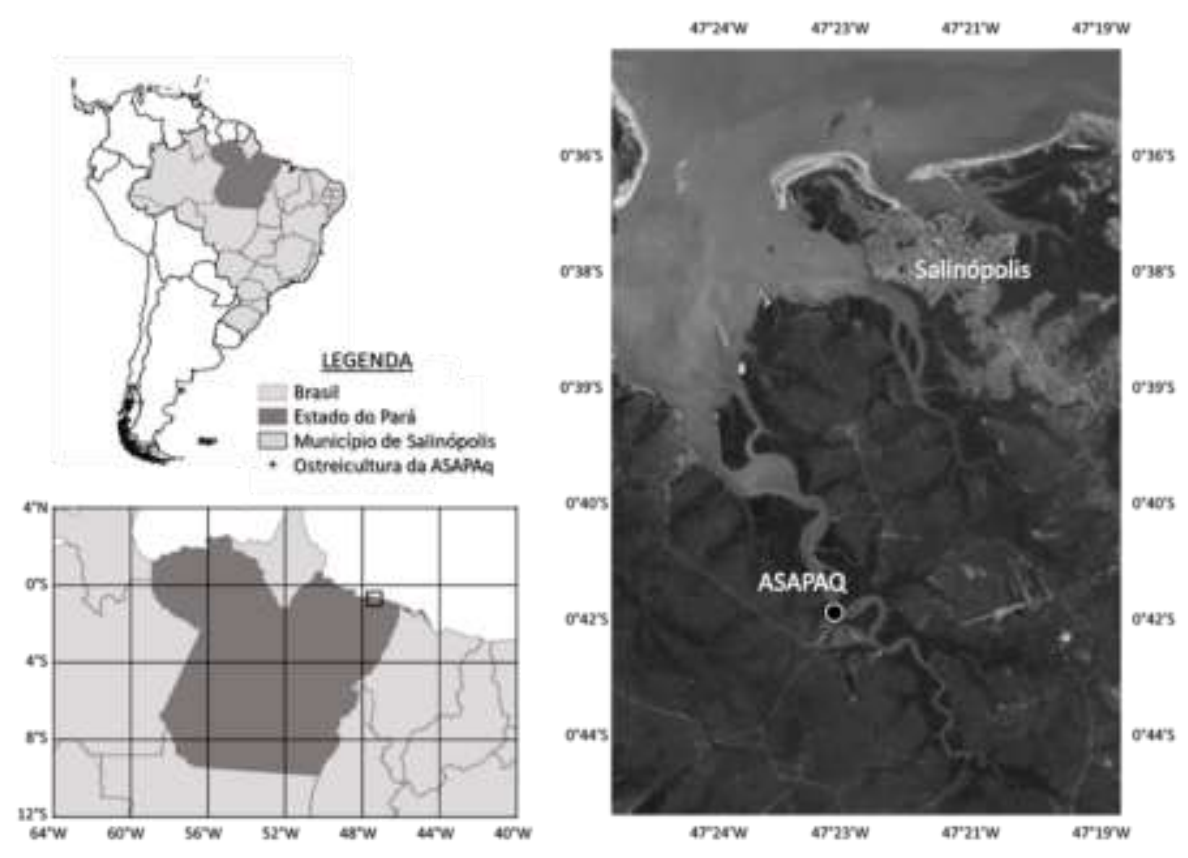

Fonte: Autores.

\section{Procedimento de amostragem}

Neste estudo realizou-se nove coletas, entre maio de 2016 e abril de 2017, amostrando-se 30 ostras/mês em lanternas previamente codificadas na ASAPAQ, para que não fossem manejadas pelos ostreicultores durante a limpeza periódica do cultivo. Ressalta-se que, as ostras coletadas representam as amostras quantitativas, sendo classificadas qualitativamente (Chagas et al., 2018; Pereira et al., 2018).

Baseou-se o processo de amostragem de acordo com Chagas (2016), realizando primeiramente a retirada do biofouling da superfície das ostras, utilizando uma escova com cerdas macias em um jato de água de baixa pressão, evitando que organismos sensíveis a choques mecânicos (e.g., poliquetas) fossem danificados. Posteriormente, triou-se o material em peneira de malha de $1 \mathrm{~mm}$, fixando os organismos em etanol 70\%. As amostras previamente codificadas foram transportadas ao Laboratório de Ecologia Bentônica Tropical da Universidade Federal Rural da Amazônia (UFRA), em Belém, para análises.

Em laboratório, realizou-se um segundo processo de triagem, que consistiu na separação dos organismos por morfotipos e fez-se a quantificação dos mesmos. Após esse processo, realizou-se a identificação taxonômica em microscópio estereoscópico, utilizando bibliografia especializada, incluindo as listas de espécies da região segundo Chagas et al. (2018) e Vale et al. (2020). 


\section{Análise dos dados}

Para a análise dos dados, primeiramente mensurou-se o comprimento total de $C$. tulipa com um paquímetro digital (TESA Data-Direct, precisão: 0,01 mm), para posteriormente, classificar as ostras de acordo com seus respectivos tamanhos comerciais: "semente" (<30 mm), "juvenil" (30-59 mm), "baby" (50-79 mm), "média" (80-100 mm) e "máster" (> $100 \mathrm{~mm})$. Em seguida, realizou-se uma análise de correlação $\left(\mathrm{R}^{2}\right)$ entre a riqueza e a abundância do biofouling encontrado e as medidas de comprimento total das ostras e entre si, com a finalidade de verificar a dependência estatística entre essas variáveis. Posteriormente, classificou-se as mesmas de acordo com o coeficiente de correlação de Pearson (r), de acordo com Rodrigues (2010). Antes de cada análise, verificou-se a normalidade dos dados com um Teste de Levene $(p=0,05)$. Em seguida, aplicouse uma Análise de Variância one-way (ANOVA one-way) para testar possíveis diferenças entre ostras de diferentes tamanhos com relação aos parâmetros riqueza e abundância do biofouling e as, realizando posteriormente um Teste de Tukey, para destacar quais variáveis diferiram entre si e para estimar o nível dessas diferenças. Todas as análises estatísticas foram realizadas a um nível de significância de 95\%, segundo Zar (2010), no software Statistica (versão 7.0).

\section{Resultados e Discussão}

Ao todo $85,2 \%(\mathrm{n}=232)$ das C. tulipa analisadas apresentaram biofouling associado à superfície das conchas (Tabela 1). No geral, encontrou-se uma riqueza de 27 espécies (dentre moluscos bivalves e gastrópodes, crustáceos e anelídeos) e abundância de 6.402 indivíduos. Do total de ostras, 27,9\% (76 ostras) apresentaram uma espécie, 21,6\% (59 ostras) duas espécies, 20,2\% (55 ostras) três espécies, 11,7\% (32 ostras) quatro espécies, 3,3\% (nove ostras) cinco espécies e apenas uma ostra com seis espécies. Além disso, $14,8 \%(\mathrm{n}=40)$ das ostras não apresentaram biofouling, sendo que destas sete foram classificadas como "semente", 10 classificadas como "juvenil", sete classificadas como "baby", 10 classificadas como "média" e seis classificadas como "máster". Ressalta-se que nenhuma semente apresentou biofouling.

Tabela 1. Número de ostras com e sem a presença de biofouling por classe de tamanho de ostras.

\begin{tabular}{ccc}
\hline Classe de tamanho & $\mathbf{N}^{\circ}$ de ostras com biofouling & $\mathbf{N}^{\circ}$ de ostras sem biofouling \\
\hline Semente & 0 & 7 \\
Juvenil & 43 & 10 \\
Média & 35 & 7 \\
Baby & 65 & 10 \\
Master & 89 & 6 \\
\hline
\end{tabular}

Fonte: Autores.

A relação entre riqueza de espécies e comprimento total de C. tulipa apresentou uma correlação baixa $\left(\mathrm{R}^{2}=0,104\right)$, tendo sido classificada como fraca $(\mathrm{r}=0,323 ; p<0,001)$. A relação entre a abundância de indivíduos e o comprimento total também apresentou uma correlação baixa $\left(\mathrm{R}^{2}=0,011\right)$ e foi classificada como nula $(\mathrm{r}=0,107 ; p=0,102)$. Do mesmo modo, a relação entre a abundância de indivíduos e a riqueza de espécies apresentou uma correlação classificada baixa $\left(R^{2}=0,092\right)$, tendo sido classificada como fraca $(\mathrm{r}=0,304 ; p<0,001)$.

Os resultados deste estudo divergem parcialmente dos encontrados por Chagas (2016), em seu estudo com C. tulipa realizados anteriormente no mesmo local, visto que a relação entre a abundância de indivíduos e a riqueza de espécies apresentou uma correlação baixa $\left(R^{2}=0,28\right)$, no entanto, de acordo com a classificação do coeficiente de correlação de Pearson, esse autor a classificou como substancial $(r=0,5)$. Essa pequena diferença deve ser analisada, pois pode indicar uma influência climática ocasionada pelo El Niño na riqueza de espécies da região, visto que o estudo realizado por Chagas (2016) ocorreu em um ano sem anomalia climática (2013) e o presente estudo em ano de El Niño (2015-2016). 
A hipótese de que a riqueza de macrobentos que compõe o biofouling associada à superfície da ostra C. tulipa difere entre as classes de tamanho comercial, foi confirmada após resultados da ANOVA one-way $\left(\mathrm{F}_{(3,228)}=11,757 ; p<0,001\right)$. Ostras de tamanho "máster" apresentam duas espécies a mais que as classificadas como "juvenil" (Tukey, $p<0,001$ ) e uma espécie a mais que aquelas classificadas como "média" (Tukey, $p<0,001$ ) (Figura 2). Em contrapartida, não houve diferenças entre os demais tamanhos comerciais analisados (Tabela 1). No entanto, a hipótese de que a abundância de macrobentos que compõe o biofouling associado à superfície da ostra difere entre as classes de tamanho comerciais, foi refutada após resultados da ANOVA one-way $\left(\mathrm{F}_{(3,228)}=1,861 ; p=0,136\right)$.

Figura 2. Médias de riqueza de espécies por classes de comprimento total ( $\mathrm{mm}$; tamanhos comerciais). Letras diferentes indicam diferenças significativas, $p<0,001$.

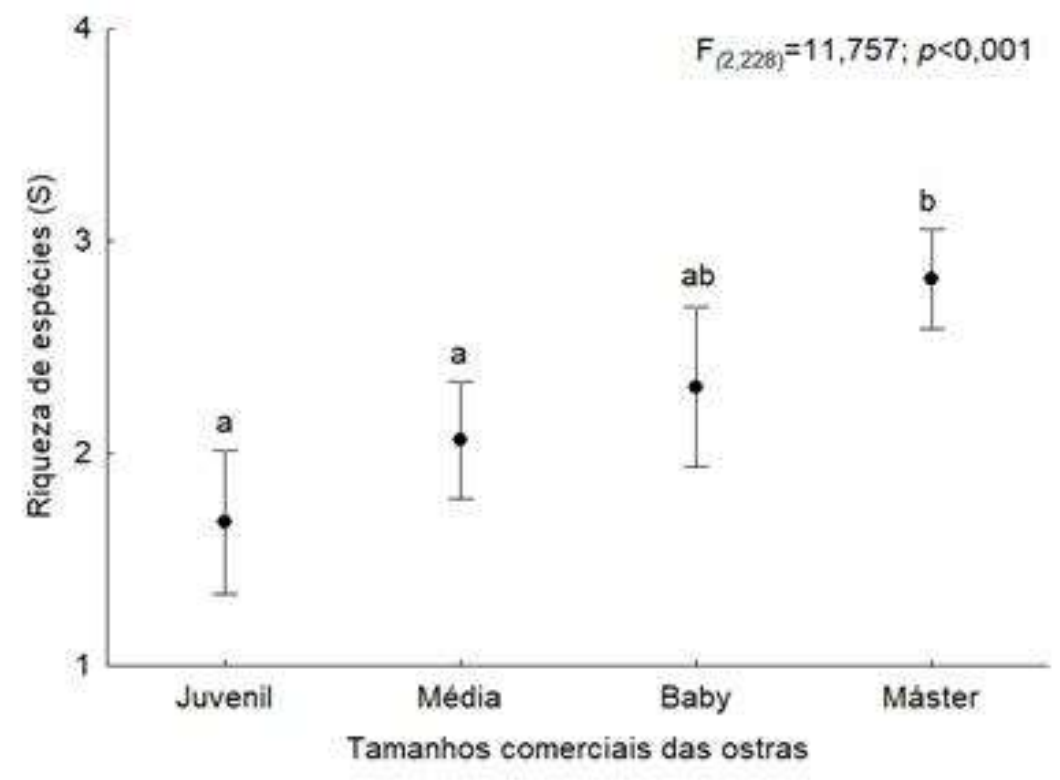

Fonte: Autores.

Tabela 2. Resultados do Teste de Tukey realizado após Anova one-way entre a riqueza de espécies e as classes de comprimento total (mm, tamanhos comerciais), apresentando o valor de $p$ e as diferenças entre riqueza de espécies (S).

\begin{tabular}{ccc}
\hline Relação & $p$-valor & Diferença entre $S$ \\
\hline "Juvenil" X "Média" & 0,296 & - \\
"Juvenil” X “Baby" & 0,059 & - \\
"Juvenil” X "Máster" & $* *$ & 2 \\
"Média" X “Máster" & $* *$ & 1 \\
"Média" X "Baby" & 0,706 & - \\
"Baby" X "Máster" & 0,108 & - \\
\hline
\end{tabular}

** Valor de $\mathrm{p}<0,001$. Fonte: Autores.

Chagas e Herrmann (2016), em seu estudo realizado no mesmo local, apresentaram resultados semelhantes ao presente, no entanto, este último indicou diferença na riqueza das ostras "média" daquelas classificadas como "juvenil" e "baby". Além disso, o resultado da relação entre a abundância e as classes de tamanho comercial foi o mesmo nos dois estudos, refutando a hipótese de que a abundância de indivíduos do biofouling associados à superfície da ostra difere entre as classes de tamanhos comerciais. Os padrões de colonização de biofouling são influenciados por diversos fatores, dentre eles: zona geográfica, características ambientais, aspectos físico-químicos, bem como suas relações alimentares intra ou interespecíficas, tipo de 
substrato, profundidade e entre outros (Bannister et al., 2019; Lacoste \& Gaertner-Mazouni, 2014; Sievers et al., 2014). No entanto, o presente estudo é o primeiro ao relacionar a colonização do biofouling à superfície do bivalve.

\section{Conclusão}

Neste estudo, confirmou-se a hipótese de que ostras maiores apresentam um maior número de espécies de biofouling quando comparadas a ostras menores. No entanto, refutou-se a hipótese de que a abundância do biofouling na superfície da ostra está relacionada ao tamanho comercial da espécie. Recomenda-se uma análise aprofundada da influência do El Niño nesse aspecto, visto que foram evidenciadas diferenças na riqueza espécies no presente estudo, realizado em ano da anomalia climática e o realizado anteriormente por Chagas e Herrmann (2016) na região.

\section{Agradecimentos}

O presente trabalho foi realizado com apoio da Coordenação de Aperfeiçoamento de Pessoal de Nível Superior CAPES, por meio de concessão de bolsa de pós-graduação ao último autor listado neste trabalho. Ressalta-se o agradecimento a Universidade Federal Rural da Amazônia (UFRA), pela disponibilidade dos espaços físicos para desenvolver o projeto. Aos membros da Associação dos Agricultores, Pecuaristas e Aquicultores - ASAPAQ, situado rio Urindeua, em nome de sua presidente D. ${ }^{a}$ Maria.

\section{Referências}

Bannister J., Sievers, M., Bush, F. \& Bloecher, N. (2019). Biofouling in marine aquaculture: a review of recent research and developments. Biofouling, 1-18. http://dx.doi.org/10.1080/08927014.2019.1640214

Camargo F. V. (2011). Biodiversidade de caranguejos Braquiúros (Crustacea: Decapoda) associada ao cultivo comercial de mexilhão Perna perna (Linnaeus, 1758) (Molusca: Bivalvia) no Litoral Norte do Estado de São Paulo. Dissertação (Mestrado em Ciências Biológicas) - Universidade Estadual Paulista, Botucatu.

Carraro J. L. F. (2008). Estrutura da comunidade de invertebrados bentônicos sésseis e suas interações com as vieiras Nodipecten nodosus no cultivo da praia do Canto Grande, Santa Catarina. Dissertação (Mestrado em Ecologia) - Universidade Federal do Rio Grande do Sul, Porto Alegre.

Chagas R. A. (2016). Biofouling no cultivo da ostra-do-mangue Crassostrea rhizophorae (Guilding, 1828) (Bivalvia: Ostreidae) em um estuário amazônico. Monografia (Bacharel em Engenharia de Pesca) - Universidade Federal Rural da Amazônia, Belém, PA. https://doi.org/10.2312/ebt.thesis.chagas.2016

Chagas R. A., Barros, M. R. F., Santos, W. C. R. \& Herrmann, M. (2018). Composition of the biofouling community associated with oyster culture in an Amazon estuary, Para state, Northern Brazil. Revista de Biologia Marina y Oceanografia, 53(1), 9-17. http://dx.doi.org/10.4067/S0718-19572018000100009

Chagas R. A. \& Herrmann, M. (2016). Abundância e diversidade de espécies que compõe o biofouling está relacionada a biomorfometria das conchas das ostras? In: XIV Seminário Anual de Iniciação Científica da UFRA, Belém - PA.

Connell S. D. \& Glasby, T. M. (1998). Do urban structures influence local abundance and diversity of subtidal epibiota? A case study from Sydney Harbous, Australia. Marine Environmental Research, 47, 373-387. http://dx.doi.org/10.1016/S0141-1136(98)00126-3

Frigotto S. F. (2011). Fauna carcinológica vágil associada às lanternas de ostreicultura na baía de Guaratuba, PR. Dissertação (Mestrado em Ciências Biológicas) - Universidade Federal do Paraná, Curitiba.

Lacoste E. \& Gaertner-Mazouni, N. (2014). Biofouling impact on production and ecosystem functioning: a review for bivalve aquaculture. Reviews in Aquaculture, 6, 1-10. https://doi.org/10.1111/raq.12063

Leite L.A. (2007). Influência da predação, parasitismo e densidade de sementes de mexilhões Perna perna (L., 1758), cultivados na Baía Norte da Ilha de Santa Catarina. Dissertação (Mestrado em Aquicultura) - Universidade Federal de Santa Catarina, Florianópolis.

Macedo P. P. B. (2012). Fauna associada ao cultivo de vieiras - Nodipecten nodosus (Linnaeus, 1758) (Mollusca, Pectinidae) - na enseada de Armação do Itapocoroy, Penha, SC. Dissertação (Mestrado em Ciências Biológicas) - Universidade Federal do Paraná, Curitiba.

Macedo P. P. B., Masunari, S. \& Corbetta, R. (2012). Crustáceos decápodos associados às cordas de cultivo do mexilhão Perna perna (Linnaeus, 1758) (Mollusca, Bivalvia, Mytilidae) na Enseada da Armação do Itapocoroy, Penha - SC. Biota Neotropica, 12(2), 185-195. http://www.biotaneotropica.org.br/v12n2/pt/abstract?inventory+bn01812022012

Marenghi F. P. \& Ozbay, G. (2010). Floating Oyster,Crassostrea virginica Gmelin 1791, Aquaculture as Habitat for Fishes and Macroinvertebrates in Delaware Inland Bays: The Comparative Value of Oyster Clusters and Loose Shell. Journal of Shellfish Research, 29(4), 889-904. http://dx.doi.org/10.2983/035.029.0422

Marenzi A. W. C. \& Branco, J. O. (2006). O cultivo do mexilhão Perna perna no município de Penha. In J. O. Branco \& A. W. C. Marenzi (Eds.), Bases ecológicas para um desenvolvimento sustentável: estudos de caso em Penha (pp. 227-244). Itajaí: Editora da UNIVALI. 
Research, Society and Development, v. 10, n. 2, e41810212475, 2021

(CC BY 4.0) | ISSN 2525-3409 | DOI: http://dx.doi.org/10.33448/rsd-v10i2.12475

Nybakken J. W. \& Bertness, M. D. (2004). Marine Biology: An ecological approach (6 ed.). São Francisco: Benjamin Cunnings.

Pereira A. S; Shitsuka D. M.; Parreira F. J. \& Shitsuka R. (2018). Metodologia da pesquisa científica UFSM.

Pinto F. M. V. S. (2007). Efeito de organismos incrustantes sobre o crescimento e a sobrevivência de ostras nativas do gênero Crassostrea em um cultivo suspenso na Baía de Guaratuba (Paraná - Brasil). Monografia (Bacharel em Oceanografia) - Universidade Federal do Paraná, Pontal do Paraná. http://hdl.handle.net/1884/36593

Rodrigues W. C. (2010). Estatística Aplicada (8 ed.). Paracambi: W.C. Rodrigues.

Shumway S. E., Davis, C., Downey, R., Karney, R., Kraeuter, J., Parsons, J., Rheault, R. \& Wikfors, G. (2003). Shellfish aquaculture in praise of sustainable economies and environments. World aquaculture, 34(4), 15-18. http://darc.cms.udel.edu/ibog/ShumwayWASarticle.pdf

Sievers M., Dempster, T., Fitridge, I. \& Keough, M. J. (2014). Monitoring biofouling communities could reduce impacts to mussel aquaculture by allowing synchronisation of husbandry techniques with peaks in settlement. Biofouling, 30(2), 203-212. http://dx.doi.org/10.1080/08927014.2013.856888

Sievers M., Dempster, T., Keough, M.J. \& Fitridge, I. (2019). Methods to prevent and treat biofouling in shellfish aquaculture. Aquaculture, 505, 263-270. http://dx.doi.org/10.1016/j.aquaculture.2019.02.071

Souza G. B. G., Passos, G. M. \& Boehs, G. (2007). Macrofauna incrustante em coletores de sururu (Mytella guyanensis) na ilha do Tanque, Península de Maraú $(B A)$. In: VIII Congresso de Ecologia do Brasil, Caxambú-MG.

Vale A. V. P., Santos, W. C. R., Barros, M. R. F., Chagas, R. A. \& Herrmann, M. (2020). Comparação de substratos artificiais na redução de bioincrustantes em um cultivo de ostras no estuário amazônico. Revista CEPSUL: Biodiversidade e Conservação Marinha, 9(e2020001), 1-16. https://www.icmbio.gov.br/revistaeletronica/index.php/cepsul/article/view/875/1142

Zar J.H. (2010). Biostatistical Analysis (5 ed.). New Jersey: Prentice Hall. 